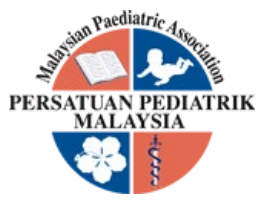

\title{
DEEP VEIN THROMBOSIS EXTENDING TO INFERIOR VENA CAVA IN A CHILD
}

\author{
Roopa B Mangshetty, Apurva A B, Mallikarjun Kobal, Aishwarya Bijapur, Kedar Gombi
}

\begin{abstract}
Deep Vein Thrombosis (DVT) is uncommon in children. We describe a case of DVT in children, highlighting importance of early diagnosis of rare disease. Here we report a case of 10-year-old boy presented with persistent pain and swelling of the left thigh. Detailed investigations led to diagnosis of DVT.
\end{abstract}

Keywords: Deep Vein Thrombosis (DVT), Inferior vena cava (IVC), Pulmonary Embolism (PE), Post Thrombotic Syndrome (PTS)

\section{Introduction}

Deep Vein Thrombosis is relatively rare in children overall incidence of $0.7 / 100,000$ paediatric population [1]. This disease is under diagnosed and under reported due to low index of suspicion among paediatricians. If identified on time and proper therapeutic measures are taken, life threatening complications like pulmonary embolism and post thrombotic syndrome like lower limb ulcer can be prevented.

\section{Case Report}

A 10-year-old boy presented with non-healing ulcer at left foot for the past 2 months which associated with fever for 3 weeks and swelling and pain of left thigh with inability to walk for 1 week.

There were no significant past medical and surgical history i.e. lower limb trauma, long bone fractures, prolonged immobilization, previous DVT or pulmonary embolism (PE). Family history revealed no significant haematological diseases in both parent's families. He has been fully immunized and had normal growth and development.

On examination, vitals were normal and peripheral pulses palpable. $A{ }^{*} 0.5 \mathrm{cms}$ ulcer irregular shaped
Received: 05 July 2020; Accepted revised manuscript: 14 September 2020

Published online: 03 December 2020

was seen on the left ankle with pale granulation tissue, pus discharge and surrounding signs of inflammation. There were also no skin changes around the wound area like spider nevus, saphena varix or any other limb deformity noted. There was obvious swelling of left thigh with erythematous, hot, tender on palpation, firm in consistency and ill-defined margins extending from groin to lower two thirds of the thigh. Decreased range of movements was around thirty degrees was noted.

Systemic examination revealed a soft and nontender abdomen with mild hepatomegaly. The liver has rounded borders and smooth surface. Cardiovascular examination was normal.

A complete blood count with peripheral smear showed normocytic normochromic blood picture with neutrophilia and raised Erythrocyte Sedimentation Rate (ESR). Serum electrolytes, renal and liver function tests were normal. Chest $X$ ray was normal and Mantoux test negative. Lipid profile revealed borderline hypertriglyceridemia and low LDL cholesterol. The Prothrombin Time

Department of Paediatrics, Mahadevappa Rampure Medical college, Kalaburagi, Karnataka

Corresponding author: Dr. Roopa B Mangshetty, Associate Professor, Department of Paediatric M.R Medical college Kalaburagi, Karnataka, India Tel: 9449069669

Email: rmangshetty@gmail.com 
(PT)/International Normalized Ratio (INR) and Activated Partial Thromboplastin Time (APTT) were normal.

A colour doppler ultrasound study and contrastenhanced computed tomography (CECT) scan of pelvis and left thigh was performed and this confirmed the diagnosis of DVT of femoral, external, internal and common iliac vein extending to inferior vena cava on left side.

An ultrasound of the abdomen showed hepatosplenomegaly and abscess with myositis in distal end of psoas muscle. Pus culture revealed Staphylococcus aureus growth.
He was started on subcutaneous Enoxaparin $1 \mathrm{mg}$ /kg twice daily for 5 days followed by oral Warfarin and INR was maintained between 2 to 3 and appropriate sensitive antibiotics was started. We followed American Society of Hematology (ASH) guidelines and used only anticoagulation therapy and did not consider IVC filter or thrombolysis in our case.

He had improved clinically and was discharged well with oral warfarin $0.1 \mathrm{mg} / \mathrm{kg}$ and dose was adjusted to maintain INR between 2-3. He was doing well on clinic follow-up and doppler ultrasound study showed signs of partial recanalization of external iliac vein.

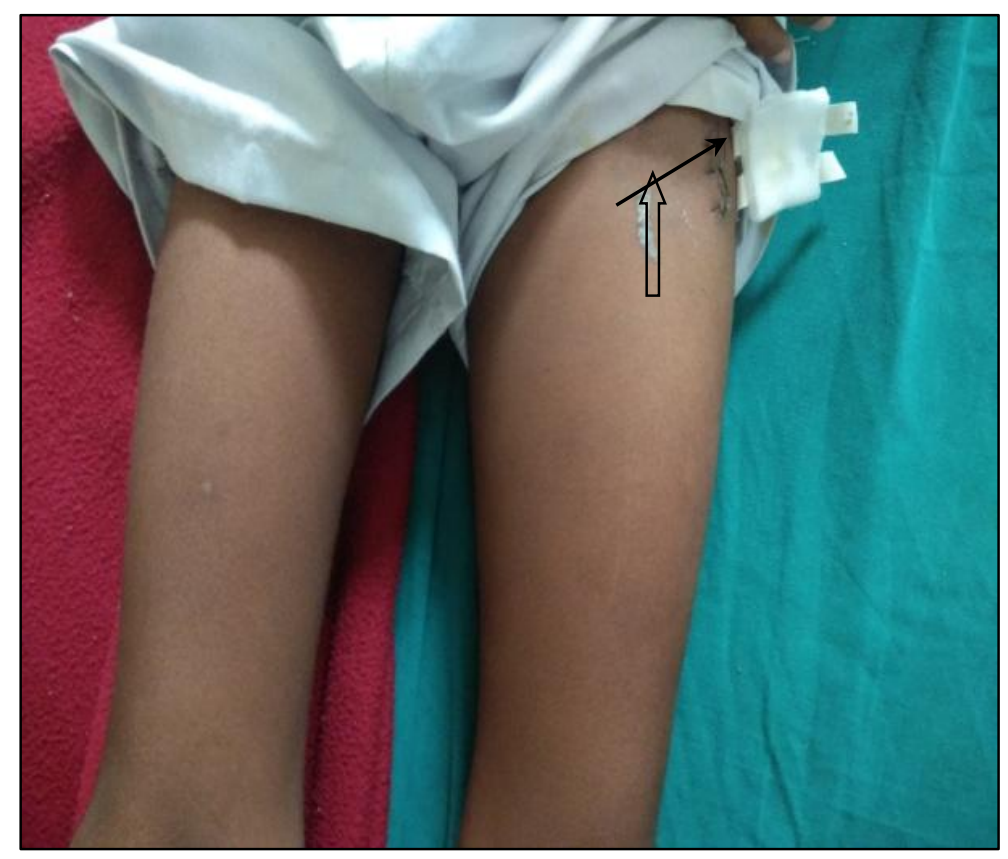

Figure 1. Swollen left leg

Left thigh circumference is $1.2 \mathrm{cms}$ more than right. 


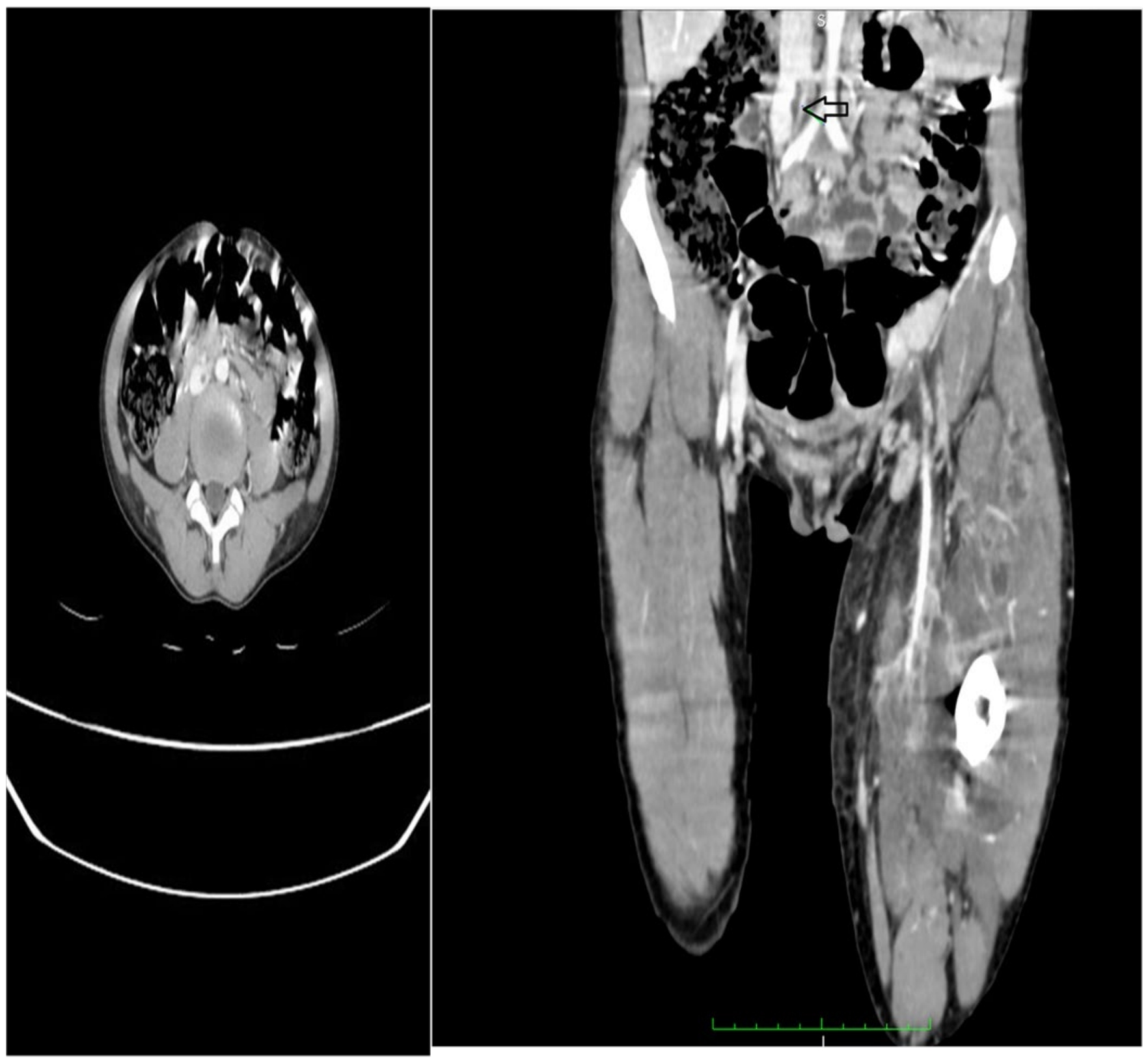

Figure 2. IVC Thrombus 


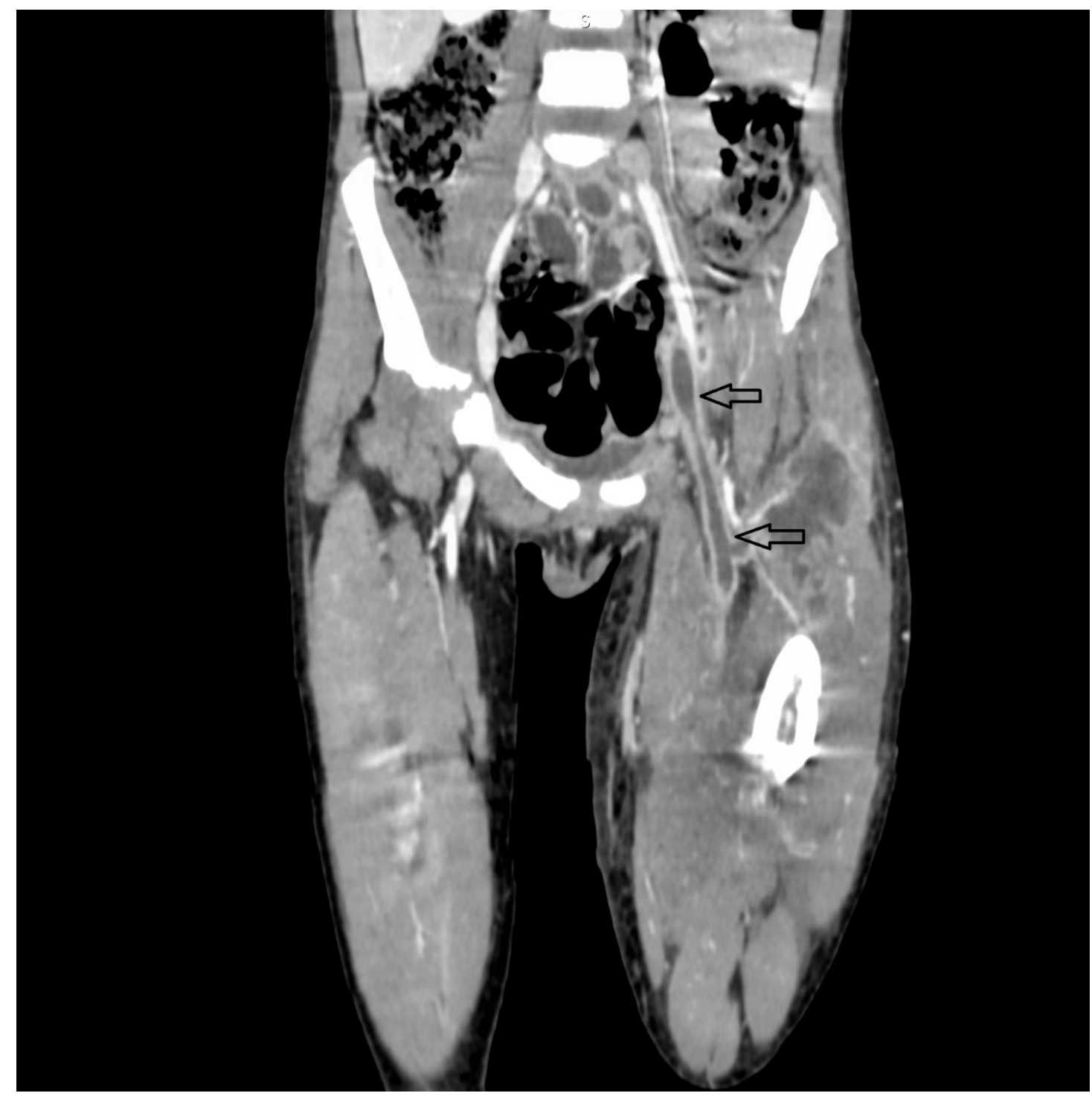

Figure 3. Extensive ileo-femoral thrombosis 


\section{Discussion}

DVT is very rare in children with overall incidence of $0.7 / 100,000$ paediatric population [1]. Majority of children who develop thromboembolism have multiple risk factors of which frequently reported among them include indwelling catheters, infection, immobility, trauma, surgery, and malignancies [2].

In our patient, infection may have contributed to development of DVT though central venous catheterization and chronic illness are the most common causes for DVT. DVT should be considered in children with unilateral swollen tender limb and local musculoskeletal focus of infection, risk of embolism and prevent recurrence. Thrombolytic therapy is reserved for patients with life or limb threatening thrombosis [1]. ASH guidelines recommend thrombolysis and IVC filter only when there is pulmonary embolism with hemodynamic compromise.

ATTRACT Trial, showed Pharmacomechanical catheter-directed thrombosis (PCDT) in acute DVT patients did not reduce the overall occurrence of post thrombotic syndrome (PTS) but did result in a higher risk of major bleeding [3]. However, in the sub-analysis for the ileo-femoral group, PCDT has clinical advantage compared to anticoagulation alone with regards to PTS prevention. Surgical bypass is rarely indicated when anticoagulation therapy is ineffective or contraindicated [1]. Our patient was treated with low molecular weight heparin and warfarin. There were signs of partial recanalization is seen up to external iliac vein during follow up.

\section{Conclusion}

DVT is rare in paediatric patients but, it does occur. Doppler ultrasonography is the first modality to diagnose DVT. Medical management is the mainstay of therapy with low molecular weight heparin for 5 days followed by oral anticoagulants up to 6 months with monitoring of INR. Adequate and early treatment can prevent thromboembolic complications.

\section{Consent}

Consent from patient's father is taken and approval by institutional ethical committee is obtained.

\section{References}

[1.] Leslie J. Raffini, J. Paul Scott. Thrombotic disorders in children. In: Richard E Behrman (Eds). Nelson textbook of pediatrics. 20th ed. Philpadelphia: Elsevier Publishing; 2016. p2394-6.

[2.] Faustino EV, Raffini LJ. Prevention of hospitalacquired venous thromboembolism in children: a review of published guidelines. Frontiers in Pediatrics. 2017 Jan 26;5:9.

[3.] Vedantham S, Goldhaber SZ, Julian JA, Kahn SR, Jaff MR, Cohen DJ, Magnuson E, Razavi MK, Comerota AJ, Gornik HL, Murphy TP. Pharmacomechanical catheter-directed thrombolysis for deep-vein thrombosis. New England Journal of Medicine. 2017 Dec 7;377(23):2240-52. 